\title{
Remarks on the First Hundred Years of Superconductivity
}

\author{
K.I. WYSOKIŃSKI* \\ Institute of Physics, M. Curie-Skłodowska University, Radziszewskiego 10, 20-031 Lublin, Poland \\ On the occasion of centenary of superconductivity discovery I recall some facts from the first period and \\ attempts to understand the phenomenon. It turns out that most famous physicists of the first half of XX century \\ have tried to solve the puzzle. Bardeen, Cooper and Schrieffer succeeded in 1957. The BCS theory successfully \\ described all known facts and offered new predictions, which soon have been confirmed experimentally contributing \\ to the widespread acceptance of the theory. It have found applications in nuclear physics, theory of neutron stars \\ and cold atomic gases. The discoveries of new superconductors in the last thirty years show that simple BCS \\ model is not enough to understand new unconventional superconductors. The studies of superconductors develop \\ vividly and still fascinate new generations of physicists working in such diverse fields as material science and string \\ theory.
}

PACS: $01.65 .+\mathrm{g}, 74.25 . \mathrm{Bt}, 74.25 . \mathrm{F}-$

The subtitle of the present XVth National School on High Temperature Superconductivity is "Hundred years of superconductivity". It is thus legitimate to recall some important facts from the first hundred years of studies of the phenomenon of superconductivity. The discovery of superconductivity in 1911 was unexpected, contrary to two years earlier liquefaction of helium. Both these great achievements were reached in the Leiden Laboratory headed by Heike Kamerlingh Onnes.

Heike Kamerlingh Onnes studied the properties of metals at low temperatures not because anybody has expected or predicted the appearance of unusual phenomena there. The mere motivation was to answer simple and controversial at the time questions of whether the resistance of metals will vanish as was expected by Drude theory. Paul Drude argued that at very low temperatures the scattering of electrons by lattice vibrations will be ineffective and the resistivity will decline with lowering temperature. On the other hand the speculative theory of Lord Kelvin (Wiliam Thomson), a very influential scientist, predicted that the resistivity should increase (in fact to infinity) at low temperatures as a result of freezing of electrons to the atoms in the material.

The vanishing of resistivity of mercury at low temperatures measured by Gilles Holst, one of the Kamerlingh Onnes' collaborators in Leiden was thus for Kamerlingh Onnes a natural and expected result. He was so sure of the result that overlooked the sudden drop of the resistance and finished the first report [1] on low temperature study of resistivity in mercury saying that the behavior is in agreement with the theory. Only after further studies inter alia of mercury doped with gold Kamerlingh Onnes realized that the completely new phenomenon is in play.

* e-mail: karol@tytan.umcs.lublin.pl
At this point it is worth to cite the remark Kamerlingh Onnes made on the same day, $8^{\text {th }}$ of April 1911, in the laboratory notebook [2]: Just before the lowest temperature [about $1.8 \mathrm{~K}$ ] was reached, the boiling suddenly stopped and was replaced by evaporation in which liquid visibly shrank. So, a remarkably strong evaporation at the surface. There is no doubt, that the superfluidity of helium was also observed on that day. But nobody in Leiden has paid any attention to that puzzling behavior of helium at low temperatures. The phenomenon of superfluidity in ${ }^{4} \mathrm{He}$ was discovered in 1937 [3], while the corresponding effect in lighter isotope ${ }^{3} \mathrm{He}$ had to wait till 1972 [4].

In the Fig. 1 the original data on temperature dependence of the resistivity in mercury measured in Leiden by Gilles Holst are reproduced. The figure was obtained in October 1911 and published by H. Kamerlingh Onnes without those who really conducted experiments. At that time it was clear that a sudden drop of resistivity has nothing to do with the ineffectiveness of phonons and marks the appearance of the completely new phenomenon of "supraconductivity".

H. Kamerlingh Onnes was a very practical man and starting the studies of resistivity $R$ in metals at helium temperatures he had in mind - besides checking if Kelvin's theory is correct - another idea of using the linear temperature dependence of $R(T)$ to build new thermometer. Even before liquefaction of helium he has observed linear behavior of $R(T)$ of many metals and basing on this linearity wanted to construct an instrument alternative to gas pressure thermometers in common use at the time [5].

H. Kamerlingh Onnes got the Nobel prize in 1913 [6] "for his investigations on the properties of matter at low temperatures which led, inter alia, to the production of liquid helium. In the Nobel lecture [6] he discussed mainly 


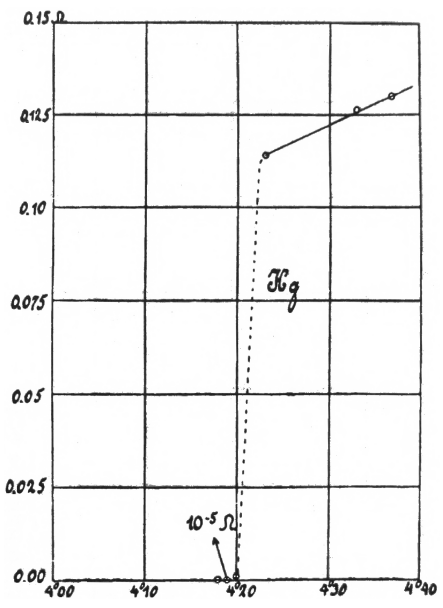

Fig. 1. The original plot showing the superconducting transition in $\mathrm{Hg}$ first published in [5]. Here it is reprinted with permission from [6]. (c) Nobel Foundation 1913.

methods which enabled to reach the goal [7] and the properties of gases but spent some time showing the resistivity of metals at low temperatures and the appearance of superconductivity in mercury.

In the Fig. 2, taken from his Nobel lecture, the dependence of the resistance is shown as function of temperature down to about $1.5 \mathrm{~K}$, roughly the lowest temperature that could be obtained in Leiden Laboratory. Discussing the curve for mercury Kamerlingh Onnes writes: As has been said, the experiment left no doubt that, as far as accuracy of measurement went, the resistance disappeared. At the same time, however, something unexpected occurred. The disappearance did not take place gradually but abruptly. From $1 / 500$ the resistance at $4.20 \mathrm{~K}$ drops to a millionth part. At the lowest temperature, $1.50 \mathrm{~K}$, it could be established that the resistance had become less than a thousand-millionth part of that at normal temperature.

During the Nobel lecture Heike Kamerlingh Onnes discussed many different studies performed in Leiden at low temperatures in cooperation with various visitors to the laboratory (e.g. Mme. Curie [came] to examine the penetrating radiation of radium). It is also worth mentioning the paragraph [6] from his Nobel lecture on the problems he encountered during the attempts to obtain very high magnetic fields with help of the superconducting coils. Shortly after the discovery of the new effect he realised that superconductors should carry large electric currents. Kamerlingh Onnes thought of using this to produce huge magnetic fields.

Being a very important and well known scientist Kamerlingh Onnes invited to Leiden other great physicist, namely Albert Einstein. Perhaps it is of interest to recall that Albert Einstein got interested in the subject and has published a paper on superconductivity on the occasion of fortieth anniversary of Kamerligh Onnes professorship in Leiden. In the paper, which is now ac-

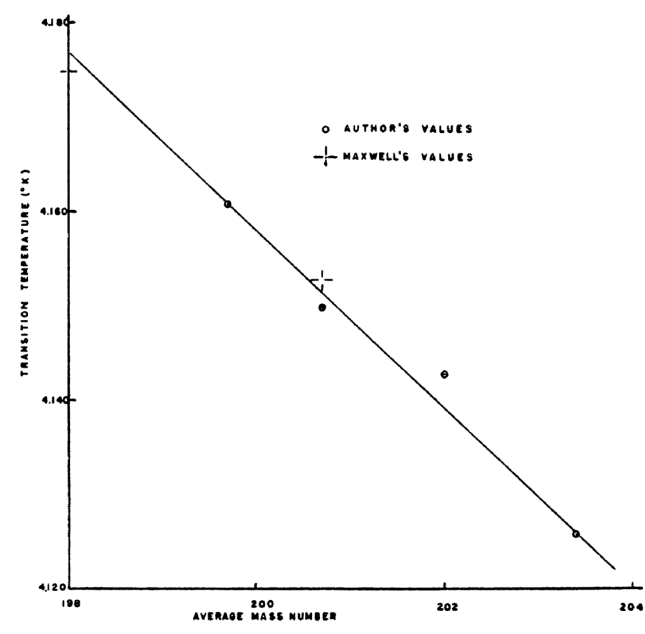

Fig. 2. The Kamerlingh Onnes plot from his Nobel lecture showing the low temperature measurements of the resistance in various metals ( $\mathrm{Au}$ of different purity, $\mathrm{Pt}$ and $\mathrm{Hg}$ ). The dashed curve shows the expectations for the pure gold. The original text describes the axis of the figure in the following way: The resistance, in fractions of the resistance at zero Centigrade, is shown as the ordinate and the temperature as the abscissa. [6]. Reprinted with permission (C)Nobel Foundation 1913.

cessible in English [8] Einstein developed the idea that supercurrents are carried through closed molecular chains where electrons undergo continuous cyclic exchanges. He also proposed an experimental verification of his theory. Namely, one has to take two different superconductors, connect them and look if the resistance of such a system in series will vanish. If the answer is affirmative then the theory is wrong as molecular chains can not extend between two different materials. Kameringh Onnes performed such experiment and falsified the Einstein's theory even before the paper appeared in print.

Probably Kamerlingh Onnes had no chance to measure, accurately enough the alternating current, which obviously flew between his biased superconductors. This current is known as and a.c. Josephson effect [9] and has been measured [10] in 1963, after it was predicted theoretically by the young student in Cambridge [11]. The successful measurements of the Josephson current was commented by one of the co-authors of the 1963 paper in such a way [11] We were able to see the effect because three conditions were satisfied: first, we knew what to look for; second, we understood what we saw. Both of these were the result of our contact with Josephson. The third condition was that we were confident of Rowell's skills in making good, clean, reliable, tunnel junctions.

Many attempts to understand the phenomenon of superconductivity by the greatest minds of the first half of the twentieth century are well known and have been discussed on many occasions [12]. That is why I shall not dwell on that. Instead, I want to spent some time by discussing two experiments, which greatly contributed to the proper understanding of the phenomenon and to 
the formulation of the correct phenomenological and later microscopic theory of superconductivity.

The first of them is the discovery in 1933 by Meissner and Ochsenfeld that the magnetic induction vanishes inside the superconductor [13]. In the "Short Note" section of the journal the authors describe their main findings on roughly half a page and summarised their two experiments saying that (translation by the author):

1. When the superconducting transition temperature was reached, the magnetic field lines have changed in the neighborhood of the superconductor in such a way as if the permeability 0 or susceptibility $-1 / 4 \pi$ were to be expected in the superconductor.

2. In the inner part of the long lead tube the magnetic field - despite the changes of it observed outside the tube according to the effect described in point 1 [...] remained nearly unchanged.

These findings which showed that the metal - superconductor transition is a reversible phenomenon allowed to apply the thermodynamic ideas and the conclusion that the entropy of the normal state is larger than that of a superconducting one and that the superconducting state is more ordered, with lower symmetry in analogy to paramagnet-ferromagnet transition. Certainly these findings have provoked Landau, Bloch and others to propose theories of superconductivity analogous to those of the ferromagnetism.

Heinz and Fritz Londons were the first to understand the real importance of the Meissner and Ochsenfeld observation and soon proposed a phenomenological theory [14] which was able to account for the observations. Fritz London correctly argued that the superconductivity is a manifestation of quantum laws on a macroscopic scale of meters or kilometers - he coined the phrase "macroscopic quantum coherence". This line of thinking was crowned with celebrated Ginzburg-Landau thermodynamic description [15] of superconductors.

The other crucial experiment I want to mention is that reporting on the isotope effect. In fact, there were two reports $[16,17]$, both received by Physical Review on the same day, 24 March 1950, and published in the same issue of the journal in section Letters to the Editor. One was written by E. Maxwell from NBS, Washington D.C. and the other one - by C.A. Reynolds and Colleagues from Rutgers University, New Jersey. Both groups were aware of their work and mutually cited each others work. It is important to note the nice agreement of those very precise and careful measurements as can be seen in the Fig. 3 reproduced from [17]. The superconducting transition temperature of natural mercury with average atomic weight 200.6 was found to be $4.156 \mathrm{~K}$ in one the papers [16] and $T_{c}=4.177 \mathrm{~K}$ for ${ }^{198} \mathrm{Hg}$. The other group [17] reported $T_{c}=4.150 \mathrm{~K}$ for natural mercury and $T_{c}=4.143$ $\mathrm{K}$ for the sample consisting of the ${ }^{202} \mathrm{Hg}$ isotope. The average slope of the line in the Fig. 3 has been estimated to be $0.009 \mathrm{~K} /$ mass number.

It is interesting to note, that only two month later the theoretical paper by H. Fröhlich proposing that the

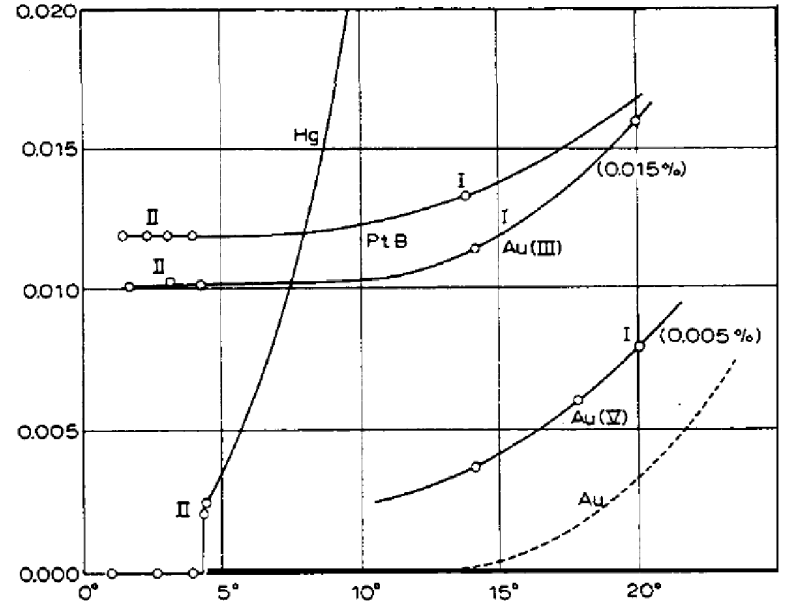

Fig. 3. The original plot [17] showing the isotope effect in Hg. Reprinted with permission. (c)(1950) by American Physical Society.

interaction between electrons and lattice vibrations was responsible for the superconductivity [18] was received by the same journal. The author mentioned the experimental papers on the isotope effect only in the "Note added in proof" with the remark: The isotope effect [...] which has recently come to my notice follows immediately from the proportionality of $|S|$ with the inverse isotopic mass $1 / M$ [...]. This agreement provides a direct check for the fundamental assumptions of the theory. In his theory Fröhlich found an effective interaction in the momentum space. As we know it from the Bardeen, Cooper and Schrieffer theory (BCS) [19] this effective attractive interaction is responsible for the superconducting instability. In the paper [18] one reads about the effective interaction: roughly speaking it is repulsive for equal energies but different directions of $\boldsymbol{k}$ and attractive otherwise. Both, the experimental data on the isotope effect and the theoretical analysis of effective interaction, together with the prediction of its attractive character contributed greatly to the formulation of the correct microscopic theory of superconductivity [19] in 1957. In this context it is important to mention that Kamerligh Onnes and Tuyn in 1922 and independently E. Justi in 1941 (see citations 1 and 2 in [17]) have checked that the superconducting transition temperature of the natural lead is the same as that of lead obtained from the decay of uranium.

In the decade after the correct microscopic description of superconductivity has been achieved [19] many new experiments were conducted which fully supported the theory. The detrimental role of magnetic impurities on the superconductivity and their robustness against nonmagnetic impurities have been explained, respectively by A.A. Abrikosov and P.W. Anderson. The strong coupling approach to the superconductivity was developed by G.M. Eliashberg and applied to strong-coupled superconductors by W.L. McMillan. As a result in the late sixties of the last century the superconductivity research 
was in decline. The famous two volume work [20] published in 1969 has summarised all known facts and theories of classic superconductors and was considered by some as a last nail to the coffin of superconductivity.

The completely unexpected discovery of superconductivity with relatively high transition temperature of about 9K in $\mathrm{PdH}_{x}$ [21] by Tadeusz Skośkiewicz in 1972 and shortly after that the negative isotope effect [22] by the same author gave new impetus to the field. The other great Polish discovery of the time, namely the coexistence of superconductivity and ferromagnetism in $\mathrm{Y}_{9} \mathrm{Co}_{7}$ (or $\mathrm{Y}_{4} \mathrm{Co}_{3}$ ) by Andrzej Kołodziejczyk [23] remained virtually unnoticed. The superconductivity in heavy fermion

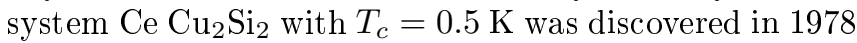
by Frank Steglich et al. [24] and still provides a puzzle [25].

The situation changed completely after the discovery of high temperature superconductivity (HTS) in La oxides by Bednorz and Müller in 1986 [26], followed by the discovery of other families of HTS with maximum transition temperatures up to $138 \mathrm{~K}$ at ambient and $166 \mathrm{~K}$ at high pressure in $\mathrm{HgBa}_{2} \mathrm{Ca}_{2} \mathrm{Cu}_{3} \mathrm{O}_{8-\delta}$. These findings evoked an unprecedented surge of experimental and theoretical activities in the field. We note by passing the 25th anniversary of HTS and the fifth anniversary of the first report [27] on the superconductivity in iron pnictides - the other class of high temperature superconductivity with highest $T_{c}=56 \mathrm{~K}$.

The numerous experimental discoveries of new superconductors during last three decades (strontium ruthenates, alkali doped fullerides, $\mathrm{MgB}_{2}$, non-centrosymmetric superconductors, etc.) have sparked new and unprecedented research activity with many new theoretical ideas and views. Unconventional superconductors are at the forefront of condensed matter physics. The microscopic mechanisms operating in those materials are at the center of scientific debate [28].

The BCS pairing theory explaining the resistance-less flow of electrons accompanied by the expulsion of the magnetic field from inside of the superconductor has influenced the way we think on various problems in physics. It has found many application in different scientific areas e.g.: it was applied (i) to atomic nuclei to explain why the even-even nuclei are more tightly bound than even-odd or odd-odd ones; (ii) to neutron stars which superfluidity may explain some properties of pulsars; (iii) the pairing may be in play in quark-gluon plasma expected to appear during heavy ion collisions.

The description of superfluidity in ${ }^{3} \mathrm{He}$ at about $2 \mathrm{mK}$ and fermionic ultra cold atoms at temperatures as low as $10^{-8} \mathrm{~K}$ require pairing of atoms. In the case of ${ }^{3} \mathrm{He}$ the quantum state is an anisotropic generalisation of the BCS original idea [29], with the same underlying principle of spontaneous breaking of the symmetry. The search for Majorana fermions - the particles which are their own antiparticles - in exotic superconductors with spin triplet chiral p-wave type of order parameter is conducted by several teams of researchers [30]. The recent connec- tions between the condensed matter physics and string theory have resulted in the new ideas and ways of approaching the pairing known as holographic superconductivity. More generally, the application of holographic methods of string theory to condensed matter physics brings a hope of accessing the strong coupling limit in the models and theories of the latter branch of physics [31].

The generalization of the spontaneous symmetry breaking from gauge symmetry to non-Abelian symmetries of interacting particles has become the most essential ingredient of the modern theory of elementary particles [32]. The so called Anderson-Higgs mechanism $[33,34]$ is expected to generate masses of particles. The notion of spontaneous symmetry breaking first encountered in the BCS theory of superconductivity has penetrated to many branches of physics and became one of the paradigms of modern physics [35].

\section{Acknowledgement}

This work has been partially supported by the Ministry of Science and Education under the grant No. N202 2631 38. I am grateful to Prof. Tadeusz Domański for encouraging me to write this paper.

\section{References}

[1] H.K. Onnes, Commun. Phys. Lab. Univ. Leiden 12, 120 (1911).

[2] D. van Delft, P. Kes, Phys. Today 63, 38 (2010).

[3] P. Kapitza, Nature 141, 74 (1937); J.F. Allen, A.D. Misener, Nature 141, 75 (1937).

[4] D.D. Osheroff, R.C. Richardson, D.M. Lee, Phys. Rev. Lett. 28, 885 (1972).

[5] H.K. Onnes, Commun. Phys. Lab. Univ. Leiden. Suppl. 29 (Nov. 1911).

[6] Heike Kamerlingh Onnes - Nobel Lecture, www.nobelprize.org/nobel_prizes/physics/laureates / 1913/onnes-lecture.html.

[7] In the Nobel lecture H. Kamerlingh Onnes on many occasions credits the work done in Krakow e.g.: Just as I was thinking about how to do this, the basic classic work of Wroblewski and Olszewski appeared on the static liquefaction of oxygen..

[8] A. Einstein, arxiv.org/abs/physics/0510251.

[9] B.D. Josephson, Phys. Lett. 1, 251 (1962).

[10] P.W. Anderson, J.M. Rowell, Phys. Rev. Lett. 10, 230 (1963).

[11] P.W. Anderson, Phys. Today 23, 23 (1970).

[12] P.H.E. Meijer, Am. J. Phys. 62, 1105 (1994); Special issue of Phys. World 24, no. 4 (2011); A. Cho, Science 332, 190 (2011); A. Mann, Nature 475, 280 (2011); L. Hoddeson, G. Baym, M. Eckert, Rev. Mod. Phys. 59, 287 (1987); D. Goodstein, J. Goodstein, Phys. Perspect. 2, 30 (2000).

[13] W. Meissner, R. Ochsenfeld, Naturwissenschaften 21, 787 (1933). 
[14] F. London, H. London, Proc. Roy. Soc. A149, 71 (1935).

[15] W.L. Ginzburg, L.D. Landau, Zh. Eksp. Teor. Fiz. 20, 1064 (1950).

[16] E. Maxwell, Phys. Rev. 78, 477 (1950).

[17] C.A. Reynolds, B. Serin, W.H. Wright, L.B. Nesbitt, Phys. Rev. 78, 487 (1950).

[18] H. Fröhlich, Phys. Rev. 79, 845 (1950).

[19] J. Bardeen, L.N. Cooper, J.R. Schrieffer, Phys. Rev. 108, 1175 (1957).

[20] In Superconductivity, Ed. R.D. Parks, Marcel Dekker, New York 1969

[21] T. Skośkiewicz, Phys. Status Solidi A 11, K123 (1972).

[22] T. Skośkiewicz, A.W. Szafranski, W. Bujnowski, B. Baranowski, J. Phys. C: Solid State Phys. 7, 2670 (1974).

[23] A. Kołodziejczyk, B.V. Sarkissian, B.R. Coles, J. Phys. F: Met. Phys. 10, L333 (1980); A. Kolodziejczyk, Physica B 130, 189 (1985).

[24] F. Steglich, H. Aarts, C. Bredl, W. Lieke, D. Meschede, W. Franz, H. Schäfer, Phys. Rev. Lett. 43, $1892(1979)$
[25] R. Doradziński, J. Spałek, Phys. Rev. B 56, R14239 (1997).

[26] K.A. Müller, J.G. Bednorz, Z. Phys. B: Condens. Matter Quanta 64, 189 (1986).

[27] Y. Kamihara, H. Hiramatsu, M. Hirano, R. Kanamura, H. Yanagi, T. Kamiya, H. Hosono, J. Am. Chem. Soc. 128, 10012 (2006).

[28] M.R. Norman, Science 332, 196 (2011); F. Wang, D.H. Lee, Science 332, 200 (2011).

[29] P.W. Anderson, J. Low Temp. Phys. 164, 119 (2011).

[30] R.F. Service, Science 332, 193 (2011).

[31] S.A. Hartnoll, Classical Quantum Gravity 26, 224002 (2009); Z. Merali, Nature 478, 302 (2011).

[32] G. Bhattacharyya, Rep. Prog. Phys. 74, 026201 (2011).

[33] P.W. Anderson, Phys. Rev. 130, 439 (1963).

[34] P. Higgs, C.R. Phys. 8, 970 (2007).

[35] A.M.J. Schakel, Boulevard of broken symmetries: effective field theories of condensed matter, World Scientific, Singapore 2008 